Communication

\title{
Polyvinyl Alcohol/Chitosan/Polyhexamethylene Biguanide Phase Separation System: A Potential Topical Antibacterial Formulation with Enhanced Antimicrobial Effect
}

\author{
Yunzhou Ni ${ }^{*}{ }^{\dagger}$, Zhixiang Qian ${ }^{\dagger}$, Yu Yin *, Weien Yuan, Fei Wu and Tuo Jin * \\ School of Pharmacy, Shanghai Jiao Tong University, Shanghai 200240, China; 820876094@sjtu.edu.cn (Z.Q.); \\ yuanweien@126.com (W.Y.); feiwu@sjtu.edu.cn (F.W.) \\ * Correspondence: yunzhouni@163.com (Y.N.); hccbyinyu@163.com (Y.Y.); tjin@sjtu.edu.cn (T.J.); \\ Tel.: +86-21-34205072 (Y.N. \& T.J.); +86-21-34204528 (Y.Y.) \\ + These authors contributed equally to this work. \\ Academic Editors: Silvia Panzavolta

Received: 25 January 2020; Accepted: 12 March 2020; Published: 15 March 2020

\begin{abstract}
An aqueous polyvinyl alcohol (PVA)/chitosan (CHT)/polyhexamethylene biguanide (PHMB) blends (PVA/CHT/PHMB blends) has been developed as a potential low dose topical antibacterial formulation with enhanced antimicrobial effect. The preparation of PVA/CHT/PHMB blends was quite facilely, with just dissolved PVA, CHT, PHMB in water in order. There was the aggregates with $100 \mathrm{~nm}$ size around induced by phase separation in the blends and an aqueous two-phase system (ATPS) formed, as non-ionic polymer PVA formed a continuous phase and cationic polymer CHT and PHMB formed dispersed phases. The minimum inhibitory concentration (MIC) of PHMB in the PVA/CHT/PHMB blends was $0.5 \mu \mathrm{g} / \mathrm{mL}$, which was four times lower than the MIC of PHMB individually. A phase separation increased zeta potential mechanism was proposed to explain the enhanced antibacterial activities. In addition, the blends could easily form film on the skin surface with good water vapor permeability and be used as a liquid bandage to accelerate the scratch wound healing process of nude mouse. These findings provide experimental evidence that the PHMB-functionalized blends could be further explored as low-dose topical antibacterial formulations, and the nano-sized phase separation strategy could be used to design novel low-dose topical antibacterial formulations with an enhanced antimicrobial effect.
\end{abstract}

Keywords: polyvinyl alcohol; chitosan; polyhexamethylene biguanide; phase separation; enhanced antimicrobial effect; low dose; topical antibacterial formulation

\section{Introduction}

Topical antibacterial agents $[1,2]$ are widely used to treat localized infections, and also can be used as prophylactic agents to prevent infection following dermatologic surgery, abrasions, cosmetic procedures. Although adverse effects of topical antibacterial agents are rare, allergic contact dermatitis is well documented [3]. Generally, side effects are dose responsive, as the incidence of side effects are fewer with low dose antibacterial therapy [4,5]. Hence, the development of novel low dose antibacterial system with effective antibacterial effects is important and necessary. Combination drugs yielding synergistic effect is an option for antibacterial therapy [6], which can reduce the dose of antibacterial agents. Therefore, it is proposed to develop a new combination strategy which could enhance antibacterial activity with low drug dose.

In recent years, nanotechnology [7] has been introduced into the antibacterial research area and achieved significant progress. Kinds of nanocarriers including micelles [8], liposomes [9], 
dendrimers [10], and nanogels [11] etc., have been developed. Generally, the fabrication process requires the preparation of nanocarriers first, and then introduce an antibacterial agent into nanocarriers via kinds of methods. However, these methods usually involve several preparation steps, and the high yield of antibacterial nanocarriers is a challenge. Therefore, it is worth developing novel facile strategies to prepare antibacterial agent containing nanocarrier systems.

Polyhexamethylene biguanide (PHMB) $[12,13]$ is one kind of cationic polymer and has broad-spectrum antibacterial activities with high therapeutic index. It has attracted wide attention as antimicrobial agent against many pathogens [14] including Gram-positive bacteria, Gram-negative bacteria, fungi and certain viruses. PHMB is relatively safe when it is used on skin, eyes, and epithelium of wounds. It can be available as disinfecting solution [15] and also can be impregnated into dressings [16]. Some recent studies reported that nanocarriers had been used to delivery PHMB to obtain better antibacterial effects. Elnaz et al. [17] prepared PHMB loaded nano cationic liposome with a smaller size $(34 \mathrm{~nm})$ and found its antibacterial activity was enhanced against Staphylococcus aureus (S. aureus) and Escherichia coli (E. coli). Sumaira et al. [18] developed PHMB-functionalized silver nanoparticles and found that its antibacterial activity was about 100 times higher than previous report. However, reports on facilely preparation PHMB-functionalized nano-systems were still few.

The objective of this study was to develop a novel facile strategy to prepare a low dose PHMB-functionalized nano-system as a potential topical antibacterial formulation with enhanced antimicrobial effect. As illustrated in Scheme 1, the fabrication process of PVA/CHT/PHMB blends was just dissolved PVA, CHT, and PHMB in water in order, which was highly simple. The low dose PHMB-functionalized blends exhibited the enhanced antibacterial effect and the phase separation between PVA and CHT and PHMB was supposed to be responsible for it. Interestingly, the blends could promote scratch wound healing of nude mouse. These aspects make this method attractive from a topical antibacterial formulation point of view.

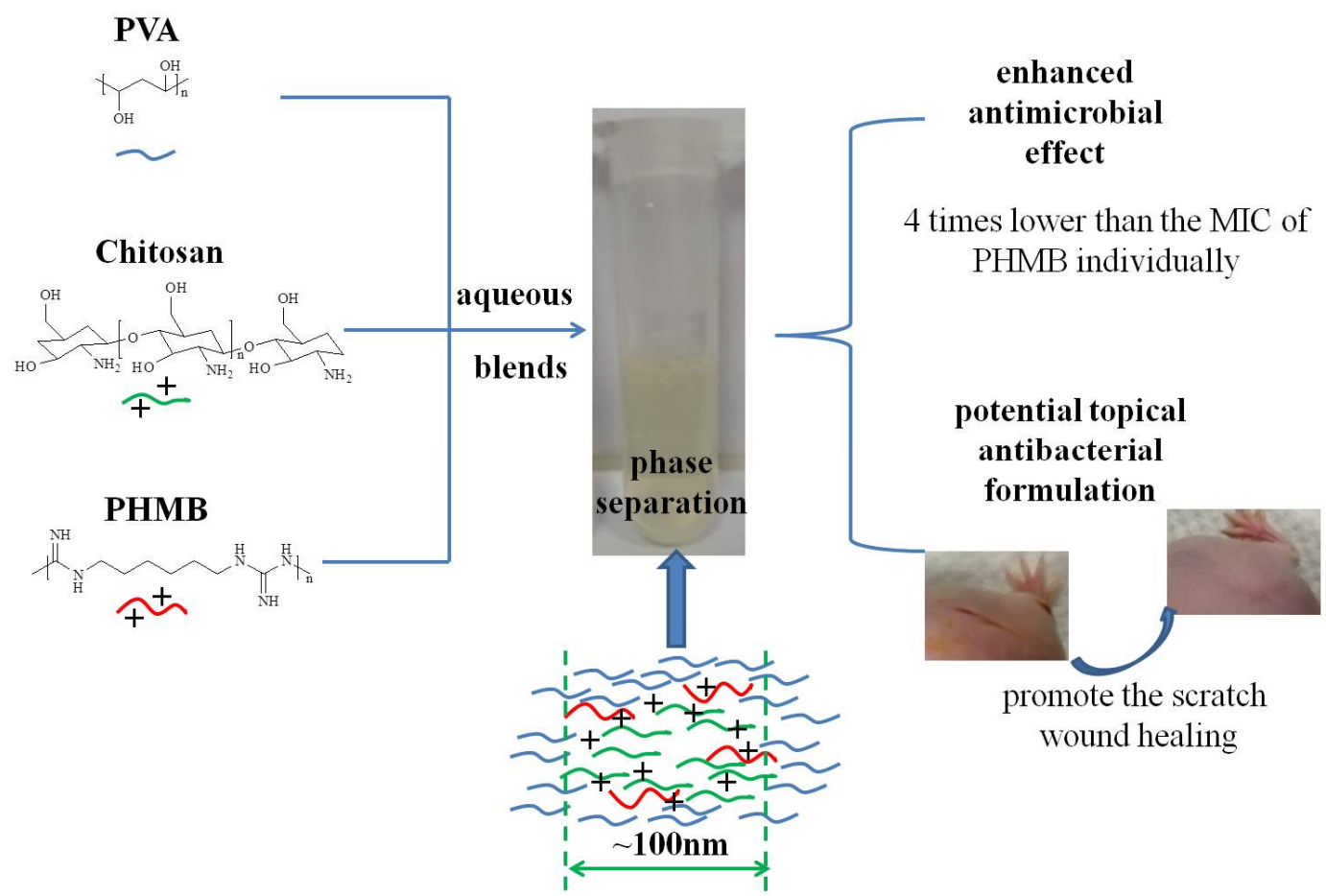

Scheme 1. Schematic illustration of preparation of polyvinyl alcohol/chitosan/polyhexamethylene biguanide blends (PVA/CHT/PHMB blends) as a potential topical antibacterial formulation with enhanced antimicrobial effect. 


\section{Materials and Methods}

\subsection{Materials}

Chitosan Hydrochloride (DAC degree 80.0-90.0\%) was as a gift from Zhejiang Golden-Shell Pharmaceutical Co. Ltd. Poly(vinyl alcohol) $\left(\mathrm{M}_{\mathrm{W}} 8000 \sim 10,000,87-89 \%\right.$ hydrolyzed) were purchased from Sigma-Aldrich (Shanghai, China). Poly(hexamethylenebiguanide) hydrochloride were purchased from Macklin Inc (Shanghai, China). The water used in all experiments was prepared using a Milli-Q purification system (Millipore Corporation, Bedford, MA, USA) and had a resistivity greater than 18.2 $\mathrm{M} \Omega \cdot \mathrm{cm}$. All other chemicals were purchased from domestic suppliers and used as received. Clear polystyrene tissue culture treated 96-well plates were obtained from Corning Costar (Corning, New York, NY, USA).

\subsection{Preparation of PVA/CHT/PHMB Blends}

PVA, CHT and PHMB were dissolved in water in order at room temperature $\left(25^{\circ} \mathrm{C}\right)$ to obtain the PVA/CHT/PHMB blends. Typically, $1 \mathrm{~g}$ PVA was firstly put into $20 \mathrm{~mL}$ water with stirring for $20 \mathrm{~min}$, and PVA (5\%) solution was obtained. Then, $600 \mathrm{mg}$ CHT was put into $20 \mathrm{~mL}$ PVA (5\%) solution with stirring for $6 \mathrm{~h}$, and PVA (5\%)/CHT (3\%) blends was obtained. $10 \mu \mathrm{g}$ PHMB was put into $20 \mathrm{~mL}$ PVA (5\%)/CHT (3\%) solution with stirring for $10 \mathrm{~min}$, and PVA (5\%)/CHT (3\%)/PHMB (0.5 $\mu \mathrm{g} / \mathrm{mL})$ blends was obtained. The preparation process of the blends was carried out at ambient condition.

\subsection{Bacterial Strains and Culture Conditions}

Two well characterized Staphylococcus aureus and Escherichia coli strains were obtained from the American Type Culture Collection. The bacteria were cultured in cation-adjusted Mueller-Hinton broth at $35 \pm 2{ }^{\circ} \mathrm{C}$. Prior to incubation with samples, the bacteria were cultured overnight in $5 \mathrm{~mL}$ of Mueller Hinton broth in a incubation shaker at $37^{\circ} \mathrm{C}, 150 \mathrm{rpm}$ until the culture reached $\mathrm{OD}_{600}$ of 1.0 , corresponding to $10^{9} \mathrm{CFU} / \mathrm{mL}$. The overnight cultures were diluted to $10^{7} \mathrm{CFU} / \mathrm{mL}$ with sterile broth.

\subsection{Assays for Antibacterial Activity}

The minimum inhibitory concentration (MIC) was determined by a microdilution method. The samples for antibacterial activity were prepared as follows: for PVA/CHT/PHMB blends, polyvinyl alcohol (PVA, 5\%), CHT (3\%) and PHMB $(1024 \mu \mathrm{g} / \mathrm{mL})$ were in order dissolved in water at room temperature with mild stirring. The plates used in the microdilution experiments were prepared by mixing $100 \mu \mathrm{L}$ bacterial Mueller-Hinton broth with $100 \mu \mathrm{L}$ sample solution. The final concentrations of tested antibacterial agents (PHMB) were: 0.25, 0.5, 1, 2, 4, 8, 16, 32, 64, 128, 256 and $512 \mu \mathrm{g} / \mathrm{mL}$ for samples prepared at $37^{\circ} \mathrm{C}$. For PVA/PHMB blends, CHT/PHMB blends, and PHMB, the sample prepared procedures were the same as the procedure of PVA/CHT/PHMB blends. Samples $(200 \mu \mathrm{L}$ each) were dispersed into the wells of the microdilution plates. The inoculated microdilution plates were covered with a plastic lid and incubated at $37^{\circ} \mathrm{C}$ for $24 \mathrm{~h}$. Bacterial growth was evaluated visually and the MIC was recorded as the lowest concentration that completely inhibits growth. The number of replicates for MIC assay was 3.

\subsection{DLS and Zeta Potential Measurement of PVA/CHT/PHMB Blends}

DLS and zeta potential measurements were performed on aqueous solutions with a Malvern Zetasizer Nano S (Malvern Instruments, Ltd, Westborough, MA, USA) equipped with a 4 mW He-Ne laser light operating at $633 \mathrm{~nm}$. All samples were measured at a scattering angle of $90^{\circ}$. The number of replicates for DLS and zeta potential measurements was 3 . 


\subsection{Rheological Measurement of PVA/CHT/PHMB Blends}

Dynamic, steady-state shear measurements were carried out on a controlled stress rotational rheometer (AR-G2, TA Instruments, New Castle, DE, USA) equipped with a $2^{\circ} 1^{\prime} 8^{\prime \prime}$ cone plate geometry (60 mm diameter, $58 \mu \mathrm{m}$ gap). The rheological measurements were conducted at $37^{\circ} \mathrm{C}$. The number of replicates for rheological measurements was 3.

\subsection{Preparation of PVA/CHT/PHMB Blends Formed Film}

Typically, the as-prepared $200 \mu \mathrm{L}$ PVA/CHT/PHMB blends was cast onto a clean glass plate with a rectangular shape with $10 \mathrm{~mm} \times 20 \mathrm{~mm}$, followed by drying at $30^{\circ} \mathrm{C}$ for $6 \mathrm{~h}$. Then, the formed film was peeled off from the glass plate carefully.

\subsection{Observation of the Surface Morphology of PVA/CHT/PHMB Blends Formed Film}

The surface morphology of PVA/CHT/PHMB blends formed film was observed under a Field Emission Scanning Electron Microscope (FEI Co., Hillsboro, OR, USA) operated at $5 \mathrm{kV}$. The samples were cut into small pieces $(5 \times 5 \mathrm{~mm})$, and coated with gold using a sputter coater under $15 \mathrm{~mA}$ for $60 \mathrm{~s}$.

\subsection{WVTR (Water Vapor Transmission Rate) of PVA/CHT/PHMB Blends Formed Film}

To determine the moisture permeability of PVA/CHT/PHMB blends formed film, WVTR was measured according to ASTM Standard (2010). Briefly, a circular piece of the specimen was mounted on the top of a plastic tube of $28 \mathrm{~mm}$ in diameter with $8 \mathrm{~mL}$ of distilled water. The tube was then placed into an incubator kept at $30{ }^{\circ} \mathrm{C}$ and $40 \pm 5 \%$ relative humidity $(\mathrm{RH})$. The WVTR was calculated by dividing the daily weight loss of water by the area of the vial opening.

\subsection{Mechanical Properties of PVA/CHT/PHMB Blends Formed Film}

A universal mechanical test machine (Instron, BAB-20MT, Trans cell Technology Inc., Shanghai, China) was used to determine the mechanical property at room temperature with a stretch velocity of $10 \% / \mathrm{min}$. Briefly, samples were cut into a rectangular shape with $10 \times 10 \times 0.077 \mathrm{~mm}$. The thickness of blends formed film was measured using a micrometer with $0.001 \mathrm{~mm}$ accuracy, and the thickness was measured at five different places for each sample.

\subsection{Scratch Wound Healing Test}

Two nude mice were used for the treatment of scratch wounds. They were allocated into two groups, one was treated with PVA/CHT/PHMB blends, another one was no treated. Each one was evaluated at 6 days. All animals were housed in a stable temperature and night/day cycle atmosphere with specific pathogen-free requirement, where the animal surgery was made. We put several cotton balls dipped in diethyl ether on the bottom of a $500 \mathrm{~mL}$ glass specimen bottle and close the bottle cap to make the bottle full of diethyl ether vapor. Then, we put the nude mouse into the bottle for anesthesia for about 10 20 seconds, a $10 \mathrm{~mm}$-long skin and $1 \mathrm{~mm}$ deep incision was made with a scalpel. One was treated with PVA/CHT/PHMB blends on the wounds, and another one was no treated. During the surgery, the nude mice were kept anesthetized to avoid them to touch the injury. The nude mice were raised in separate cages after surgery. Observations and procedures were performed at 1, 2, 3, 4, 5 and 6 days respectively. Animal care and use were in accordance with the guidelines of the Animal Ethics Committee for Shanghai Jiao Tong University. It has been approved by experimental animal management committee, academic committee and ethics and use committee of Shanghai Jiaotong University (Approval No. A2017073). 


\section{Results and Discussion}

\subsection{The Preparation of Blends}

The blends were obtained via dissolving PVA, CHT and PHMB in water in order at room temperature with mild stirring. Herein, we studied the physical chemical properties of the blends. Usually, viscosity of the fluids was a function of the deformability and packing of the micro-particles or aggregates [19]. The viscosity would decrease with the increasing of shearing rate because the aggregates were disintegrated gradually. Figure 1 a showed the curves (obtained at $37^{\circ} \mathrm{C}$ ) of viscosity

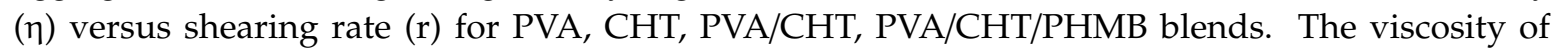
PVA/CHT/PHMB and PVA/CHT blends was significantly higher than PVA and CHT solution, and showed shear thinning behavior with the increase of shearing rate. Thus, the shear thinning behavior indicated that the aggregates had formed in the blends and could be disintegrated. In addition, the dynamic laser light scattering (DLS) data (Figure 1b) showed the aggregation with $100 \pm 30 \mathrm{~nm}$ size in PVA/CHT blends and $120 \pm 40 \mathrm{~nm}$ size in PVA/CHT/PHMB blends respectively, which further indicated that the phase separation occurred in the blends. Furthermore, the SEM image (Figure 1c) showed $130 \pm 50 \mathrm{~nm}$ size nano-phase by statistical calculation of 50 aggregations from Figure 1c in PVA/CHT/PHMB blends, which solid disclosed that the phase separation had occurred.

It was reported that polymer-polymer system could form large aggregates and separate between two different phases because of steric exclusion [20]. In PVA/CHT, PVA/CHT/PHMB blends, CHT and PHMB were hydrochloride salt, which could absorb large amounts of water and induce steric exclusion between non-ionic polymer PVA and cationic polymer CHT and PHMB. Besides steric exclusion, the interaction like H-bonding between $\mathrm{CHT}$ and PHMB acted as attractive force to enable CHT and PHMB self-assemble together and formed CHT and PHMB aggregation. Thus, the phase separation occurred in the blends and aqueous two-phase system formed, non-ionic polymer PVA formed continuous phase and cationic polymer CHT and PHMB formed dispersed phase. Obviously, the charge density of CHT and PHMB phase would increase in the ATPS accordingly. As evidence (Figure 1d), the zeta potential of PVA/CHT/PHMB blends $(32 \pm 3 \mathrm{mV})$ and PVA/CHT $(22 \pm 2 \mathrm{mV})$ blends were higher than those of CHT $(7 \pm 1 \mathrm{mV})$ solution. In addition, because the phase separation occurs spontaneously, the fabrication process of the blends would be energy saving and the obtained blends would be stable, which would be beneficial to the long-term preservation. Overall, the above results strongly demonstrated that the $100 \mathrm{~nm}$ size around phase separation with increased zeta potential had occurred in the blends. 

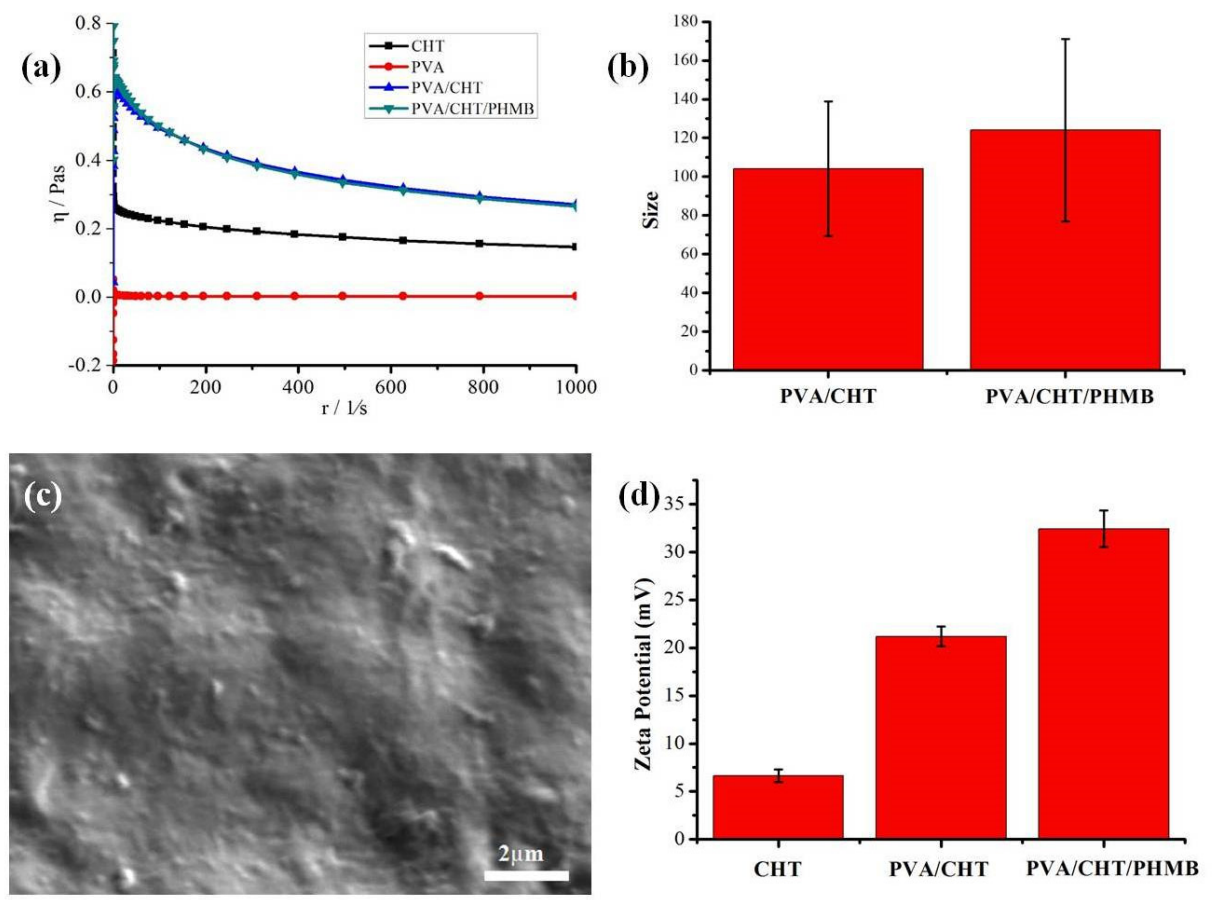

Figure 1. (a) The viscosity versus the shearing rate for PVA, CHT, PVA/CHT, PVA/CHT/PHMB solution at $37^{\circ} \mathrm{C},(\mathbf{b})$ The size of PVA/CHT and PVA/CHT/PHMB respectively, (c) The SEM images of PHMB in $\mathrm{PVA} / \mathrm{CHT}$, and (d) The zeta potential of CHT, PVA/CHT and PVA/CHT/PHMB respectively.

\subsection{The Antibacterial Activity of Blends}

In general, the antibacterial activity of chitosan nanoparticles [21] are higher than that of chitosan due to the higher surface charge density of chitosan nanoparticles. Therefore, the PVA/CHT/PHMB blends with high zeta potential was proposed to exhibit enhanced antibacterial activity. In this study, the order of dissolution was designed to dissolve PVA, CHT, PHMB orderly in order to ensure that PHMB could be assembled on the outer surface of the CHT aggregates, so that PHMB could exert its antibacterial effect. The antibacterial activity of PHMB, PVA/PHMB, CHT/PHMB, PVA/CHT/PHMB blends were compared respectively by MIC assay for evaluating the antibacterial performance [22]. Figure 2 showed the photograph of MIC assay of the samples against S. aureus and E. coli strains, and Table 1 showed the data of MIC assay. The MIC values of PHMB in the PVA/CHT/PHMB blends was 4 times lower than those of PHMB individually, which clearly indicated that the antibacterial activity of PVA/CHT/PHMB blends was significantly higher than that of PHMB. In addition, the antibacterial activity of PVA/CHT/PHMB blends was better than that of CHT/PHMB because the MIC values of PHMB in the PVA/CHT/PHMB blends was 2 times lower than those of CHT/PHMB. As the MIC values of PHMB in the PVA/CHT/PHMB blends was $0.5 \mu \mathrm{g} / \mathrm{mL}$ only, the blends as a low dose PHMB -functionalized nano-system well exhibited its enhanced antimicrobial effect.

Table 1. The data of MIC assay.

\begin{tabular}{ccccc}
\hline MIC $(\mu \mathrm{g} / \mathrm{mL})$ & PHMB & PVA/PHMB & CHT/PHMB & PVA/CHT/PHMB \\
\hline S. aureus & 2 & 2 & 1 & 0.5 \\
E. coli & 2 & 1 & 1 & 0.5 \\
\hline
\end{tabular}


(a)

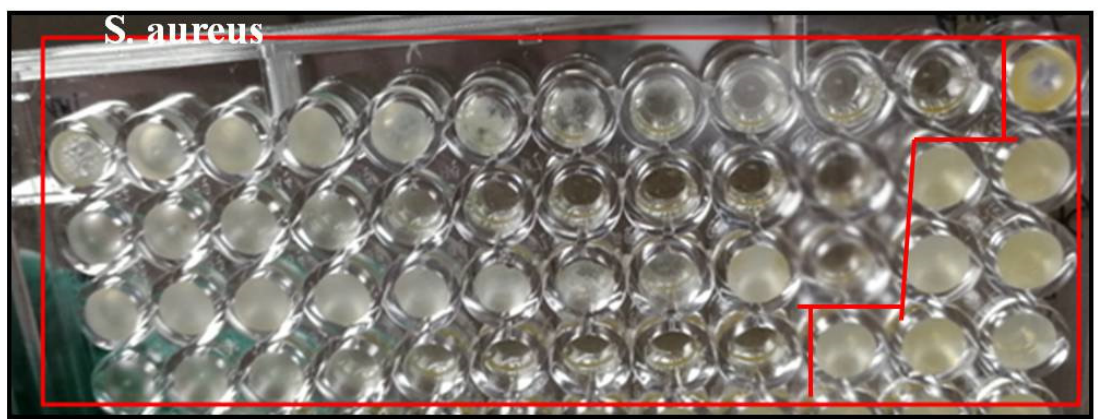

$\mathrm{PVA} / \mathrm{CHT} / \mathrm{PHMB}$

$\mathrm{PVA} / \mathrm{PHMB}$

CHT/PHMB

PHMB

(b)

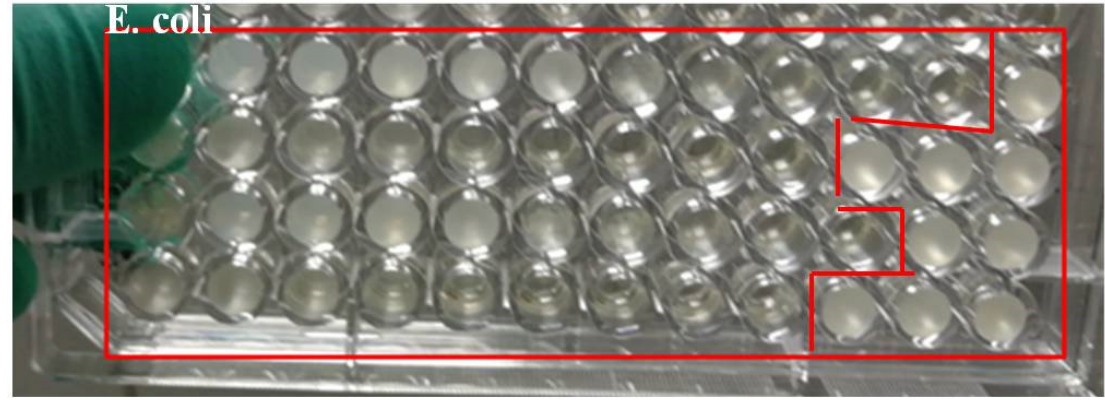

$\mathrm{PVA} / \mathrm{CHT} / \mathrm{PHMB}$

PVA/ PHMB

CHT/PHMB

PHMB

Figure 2. Photograph of minimum inhibitory concentration (MIC) of PHMB, PVA/PHMB, CHT/PHMB, $\mathrm{PVA} / \mathrm{CHT} / \mathrm{PHMB}$ in against (a) Staphylococcus aureus, and (b) Escherichia coli strains.

Such better antibacterial activity of PVA/CHT/PHMB blends was related with its high zeta potential. The characterization of high zeta potential meant that a number of high positive charge density of CHT/PHMB aggregation was in the blends. Thus, the CHT/PHMB aggregation had stronger affinity with negative charged bacteria cells membrane, and could be tightly adsorbed onto the surface of the bacteria cells, which would disturb the membrane structure and lead to the leakage of intracellular components and efficiently kill the bacteria cells. As the high zeta potential of the PVA/CHT/PHMB blends was originated from the phase separation between PVA and CHT and PHMB. Hence, a phase separation increased zeta potential mechanism (Scheme 2) was proposed to explain the enhanced antibacterial activities of the PVA/CHT/PHMB blends with low dose PHMB.

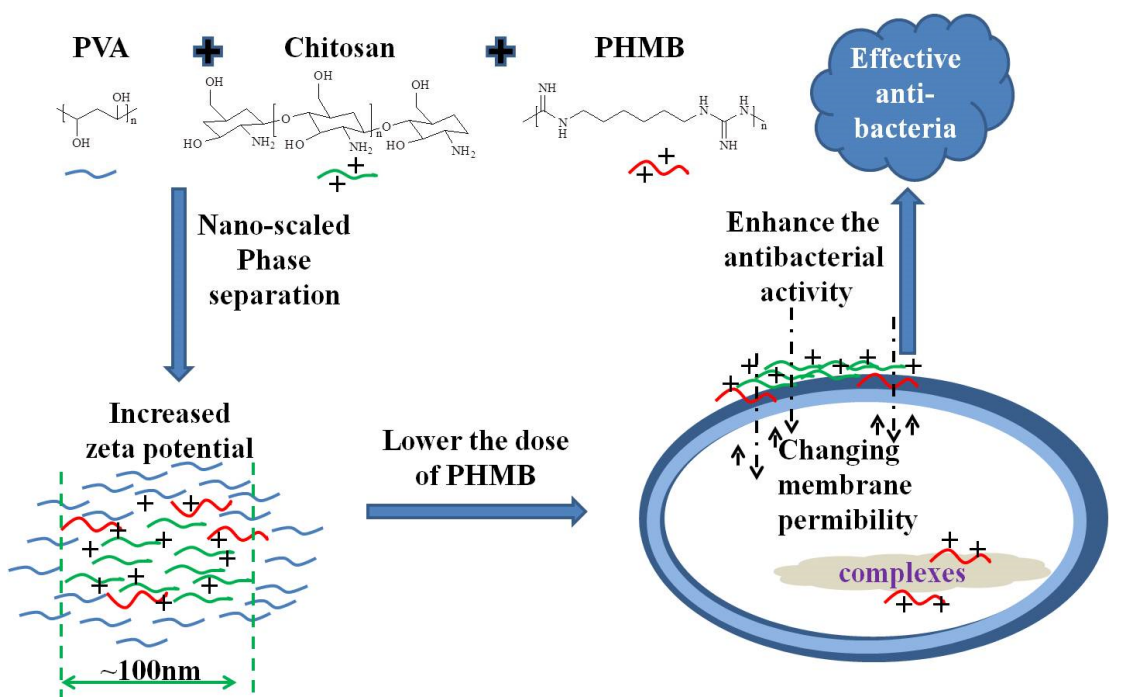

Scheme 2. Proposed mechanism of the enhanced antibacterial activity of blends. 


\subsection{PVA/CHT/PHMB Blends Film Forming Characterization}

Figure 3a clearly showed that the PVA/CHT/PHMB blends could easily form uniform and transparent films on the surface of human skin, and the film could be easily removed. Figure $3 b, c$ showed digital and optical photograph of PVA/CHT/PHMB film removed from the surface of human skin. The skin wrinkle could be clearly seen on the film, which indicated that the film could adhere on the surface of human skin well. The SEM image of the PVA/CHT/PHMB blends formed film was presented in Figure 3d, and clearly showed plenty of $100 \mathrm{~nm}$ size around nano-phase in the film, which was correspondent to the phase separation in PVA/CHT/PHMB blends showed in Figure 1. The inherent nano-scaled phase enabled the film to obtain enhanced antibacterial ability. Hence, the above data indicated that the PVA/CHT/PHMB blends would be comfortably used in different parts of the body having kinds of size, shapes and contours, especially could be used in the joints including knees and elbows, and its transparent property could allow convenient inspection of the status of wound without removing it.
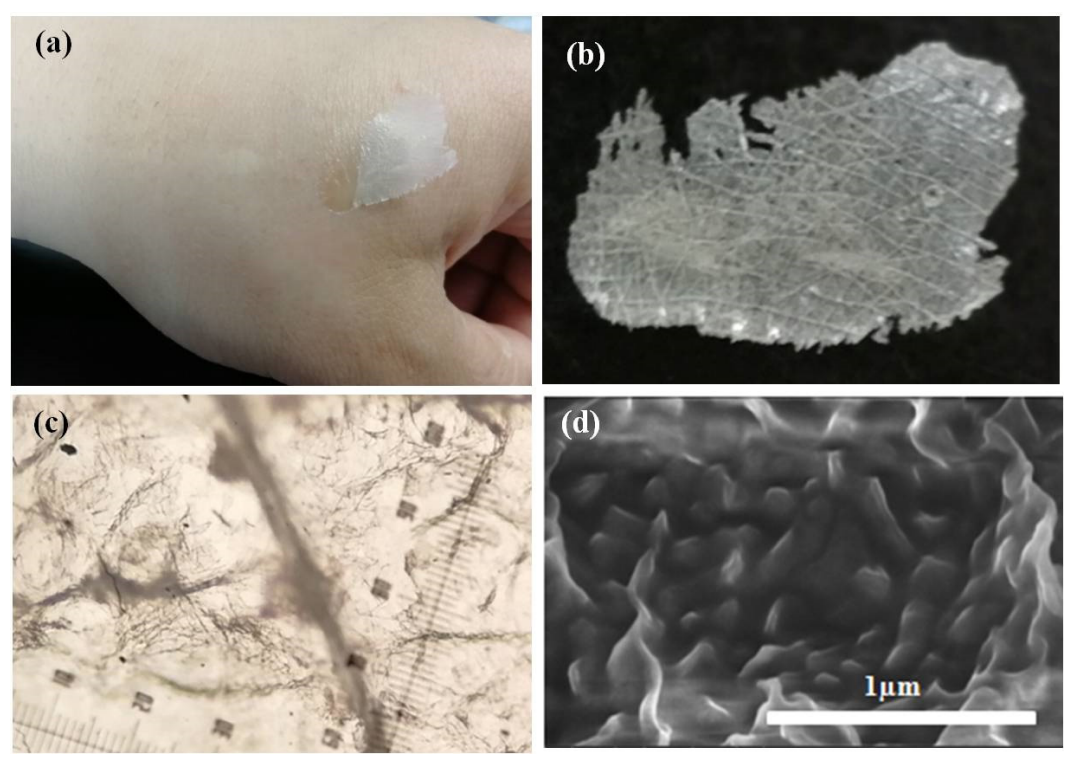

Figure 3. The appearance and properties of L-Bandage formed film. (a) Digital photograph of PVA/CHT/PHMB film on the surface of human skin; (b) digital photograph, (c) optical photograph, (d) SEM micrograph of PVA/CHT/PHMB film removed from the surface of human skin.

\subsection{WVTR (Water Vapor Transmission Rate) of PVA/CHT/PHMB Blends Formed Film}

WVTR is one of key parameters for wound dressings and proper WVTR is quite beneficial to wound healing [23]. The WVTR for the PVA/CHT/PHMB blends formed film was approximately $2000 \mathrm{~g} / \mathrm{m}^{2} /$ day (Figure 4), which indicated that the film could keep a moist environment around the wound and could support the cell growth and regeneration. The PVA/CHT/PHMB blends formed film had similar WVTR values as the wound dressings reported in the previous studies [23]. 


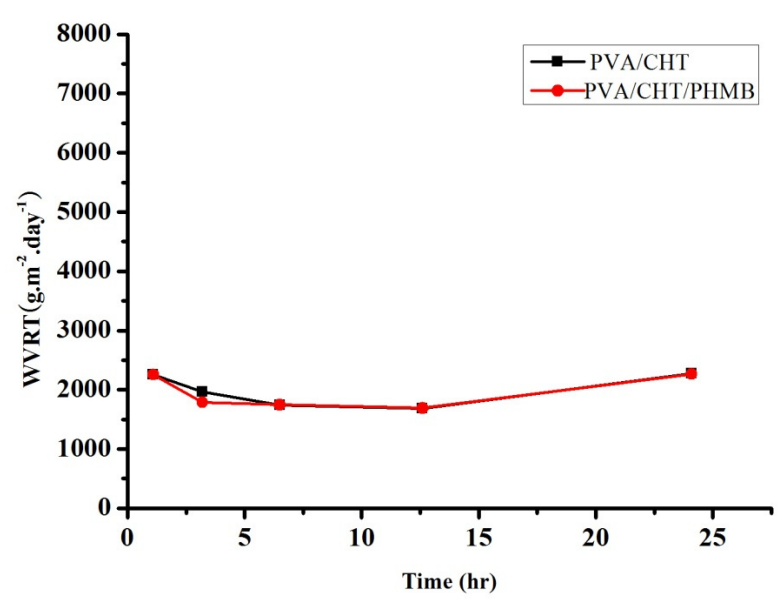

Figure 4. The WVTR of PVA/CHT/PHMB blends formed film.

\subsection{Mechanical Properties of PVA/CHT/PHMB Blends Formed Film}

The strain and stress were used to evaluate the mechanical properties of the PVA/CHT/PHMB blends formed film. We prepared PVA/CHT/PHMB blends formed film with length (10 mm), width $(10 \mathrm{~mm})$ and thickness $(0.077 \mathrm{~mm})$ to evaluate the mechanical property. The film exhibited good strength with $34 \mathrm{MPa}$ around, and the strain of film was $4 \%$ around (Figure 5). Thus, the proper WVTR and mechanical property enabled the PVA/CHT/PHMB film to be possibly used as liquid bandage for wound healing, such as abrasion and tears caused by mechanical injuries.

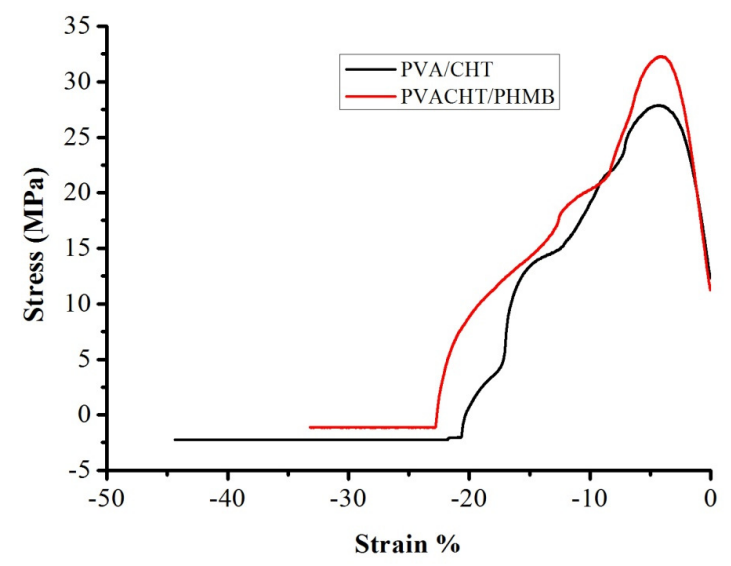

Figure 5. Strain versus strength of PVA/CHT/PHMB blends formed film.

\subsection{Scratch Wound Healing Test}

The nude mice were used to evaluate PVA/CHT/PHMB blends for the treatment of scratch wounds. The wound healing kinetics was characterized by wound appearance of wound closure, and the photographs of wound closure were provided in Figure 6. Compared with the control group (Figure $6 \mathrm{~g}-1$ ), the scratch wound was joining up better and was healed nicely after the wound was treated by PVA/CHT/PHMB blends for six days (Figure 6a-f). However, the scratch wound of the control group was not healed after six days. Therefore, the process of wound healing treated by PVA/CHT/PHMB blends was accelerated compared with control group, suggesting the PVA/CHT/PHMB blends could promote the scratch wound healing. In addition, due to the enhanced antimicrobial effect, water vapor permeability, flexible and transparent property of the blends, it was reasoned that the blends could potentially be used to treat localized infections, prevent infection following dermatologic surgery, abrasions, cosmetic procedures, and promote the wound healing. 


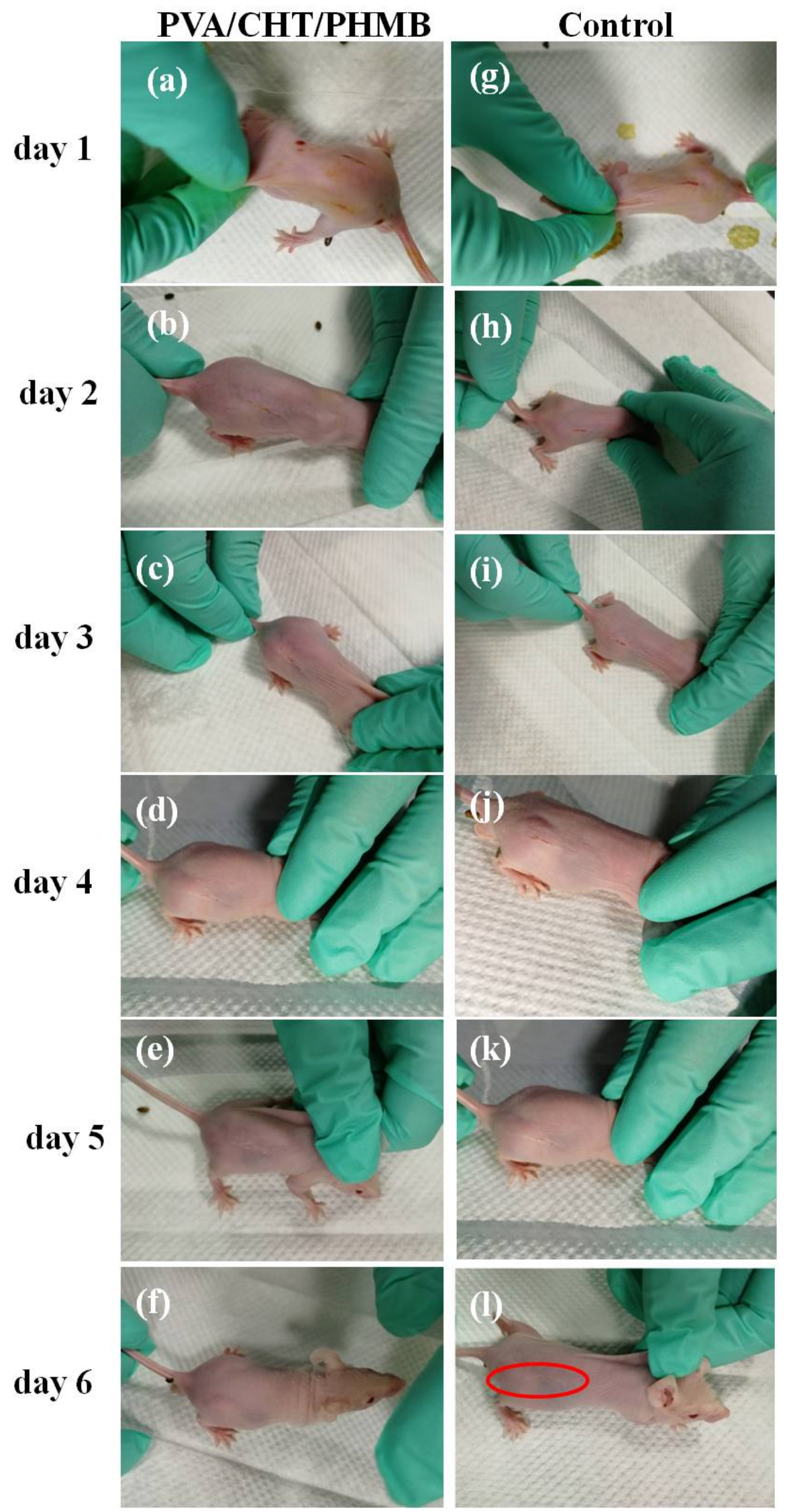

Figure 6. Scratch wound healing test in nude mouse. Compared with the control group (Figure $6 \mathbf{g}-\mathbf{1}$ ). 


\section{Conclusions}

In summary, a PVA/CHT/PHMB phase separation system has been successfully developed as a potential low dose PHMB-functionalized nano-topical antibacterial formulation with enhanced antimicrobial effect. The fabrication method was highly simple, just dissolved PVA, CHT, PHMB in water in order. There was the aggregates with size of $100 \mathrm{~nm}$ around induced by phase separation in the PVA/CHT/PHMB blends and an aqueous two-phase system formed, non-ionic polymer PVA formed continuous phase and cationic polymer $\mathrm{CHT}$ and PHMB formed dispersed phases. The MIC of PHMB in the blends was $0.5 \mu \mathrm{g} / \mathrm{mL}$, which was four times lower than the MIC of PHMB individually. A phase separation increased zeta potential mechanism was proposed to explain the enhanced antibacterial activity of the blends. The blends could easily form film on the surface of skin with good water vapor transmittance rate and mechanical properties, and be used as liquid bandage to promote the scratch wound healing of the nude mouse. The PVA/CHT/PHMB blends could be potentially applied as a low dose topical antibacterial formulation with enhanced antimicrobial effect. Furthermore, the nano-sized phase separation strategy could be further explored as antibacterial system in medicine, especially in developing novel low dose topical antibacterial formulations.

Author Contributions: Conceptualization, Y.N., Z.Q. and T.J.; formal analysis, Y.N. and Z.Q.; investigation, Y.N., Z.Q. and Y.Y.; writing-original draft preparation, Y.N.; writing—review and editing, Y.N. and T.J.; project administration, Y.N. and T.J.; funding acquisition, Y.N., W.Y., F.W. and T.J. All authors have read and agreed to the published version of the manuscript.

Funding: This research received no external funding.

Acknowledgments: We would like to thank Daijie Chen (School of Pharmacy, Shanghai Jiao Tong University) and Zhixiang Cai, Pengguang Wang (School of Chemistry and Chemical Engineering, Shanghai Jiao Tong University) for their valuable discussion and kind help.

Conflicts of Interest: The authors declare no conflict of interest.

\section{References}

1. Lio, P.A.; Kaye, E.T. Topical antibacterial agents. Infect. Dis. Clin. N. Am. 2009, 23, 945-963. [CrossRef]

2. Spann, C.T.; Tutrone, W.D.; Weinberg, J.M.; Scheinfeld, N.; Ross, B. Topical antibacterial agents for wound care: A primer. Dermatol. Surg. 2003, 29, 620-626. [PubMed]

3. Gehrig, K.A.; Warshaw, E.M. Allergic contact dermatitis to topical antibiotics: Epidemiology, responsible allergens, and management. J. Am. Acad. Dermatol. 2008, 58, 1-21. [CrossRef] [PubMed]

4. Jacobson, L.; Chabal, C.; Brody, M.C. A dose-response study of intrathecal morphine: Efficacy, duration, optimal dose, and side effects. Anesth. Analg. 1988, 67, 1082-1088. [CrossRef] [PubMed]

5. Abd-Allah, A.R.; Aly, H.A.; Moustafa, A.M.; Abdel-Aziz, A.A.; Hamada, F.M. Adverse testicular effects of some quinolone members in rats. Pharmacol. Res. 2000, 41, 211-219. [CrossRef]

6. Cottarel, G.; Wierzbowski, J. Combination drugs, an emerging option for antibacterial therapy. Trends Biotechnol. 2007, 25, 547-555. [CrossRef]

7. Jackman, J.A.; Yoon, B.K.; Li, D.; Cho, N.J. Nanotechnology Formulations for Antibacterial Free Fatty Acids and Monoglycerides. Molecules 2016, 21, 305. [CrossRef]

8. Gong, J.; Chen, M.; Zheng, Y.; Wang, S.; Wang, Y. Polymeric micelles drug delivery system in oncology. J. Control. Release 2012, 159, 312-323. [CrossRef]

9. Zylberberg, C.; Matosevic, S. Pharmaceutical liposomal drug delivery: A review of new delivery systems and a look at the regulatory landscape. Drug Deliv. 2016, 23, 3319-3329. [CrossRef]

10. Nanjwade, B.K.; Bechra, H.M.; Derkar, G.K.; Manvi, F.V.; Nanjwade, V.K. Dendrimers: Emerging polymers for drug-delivery systems. Eur. J. Pharm. Sci. 2009, 38, 185-196. [CrossRef]

11. Chacko, R.T.; Ventura, J.; Zhuang, J.; Thayumanavan, S. Polymer nanogels: A versatile nanoscopic drug delivery platform. Adv. Drug Deliver. Rev. 2012, 64, 836-851. [CrossRef] [PubMed]

12. Tan, M.; Mordiffi, S.Z.; Lang, D. Effectiveness of polyhexamethylene biguanide impregnated dressing in wound healing: A systematic review protocol. JBI Database System Rev. Implement. Rep. 2016, 14, 76-83. [CrossRef] [PubMed] 
13. Chindera, K.; Mahato, M.; Sharma, A.K.; Horsley, H.; Kloc-Muniak, K.; Kamaruzzaman, N.F.; Kumar, S.; McFarlane, A.; Stach, J.; Bentin, T.; et al. The antimicrobial polymer PHMB enters cells and selectively condenses bacterial chromosomes. Sci. Rep. 2016, 6, 23121. [CrossRef] [PubMed]

14. Hubner, N.O.; Kramer, A. Review on the efficacy, safety and clinical applications of polihexanide, a modern wound antiseptic. Skin Pharmacol. Physiol. 2010, 23, 17-27. [CrossRef]

15. Oule, M.K.; Azinwi, R.; Bernier, A.M.; Kablan, T.; Maupertuis, A.M.; Mauler, S.; Nevry, R.K.; Dembele, K.; Forbes, L.; Diop, L. Polyhexamethylene guanidine hydrochloride-based disinfectant: A novel tool to fight meticillin-resistant Staphylococcus aureus and nosocomial infections. J. Med. Microbiol. 2008, 57, 1523-1528. [CrossRef]

16. Sibbald, R.G.; Coutts, P.; Woo, K.Y. Reduction of bacterial burden and pain in chronic wounds using a new polyhexamethylene biguanide antimicrobial foam dressing-clinical trial results. Adv. Skin Wound Care 2011, 24, 78-84. [CrossRef]

17. Ahani, E.; Montazer, M.; Toliyat, T.; Mahmoudi Rad, M.; Harifi, T. Preparation of nano cationic liposome as carrier membrane for polyhexamethylene biguanide chloride through various methods utilizing higher antibacterial activities with low cell toxicity. J. Microencapsul. 2017, 34, 121-131. [CrossRef]

18. Ashraf, S.; Akhtar, N.; Ghauri, M.A.; Rajoka, M.I.; Khalid, Z.M.; Hussain, I. Polyhexamethylene biguanide functionalized cationic silver nanoparticles for enhanced antimicrobial activity. Nanoscale Res. Lett. 2012, 7, 267. [CrossRef]

19. Cai, Z.; Zhang, H.; Wei, Y.; Wu, M.; Fu, A. Shear-thinning hyaluronan-based fluid hydrogels to modulate viscoelastic properties of osteoarthritis synovial fluids. Biomater. Sci. 2019, 7, 3143-3157. [CrossRef]

20. Iqbal, M.; Tao, Y.; Xie, S.; Zhu, Y.; Chen, D.; Wang, X.; Huang, L.; Peng, D.; Sattar, A.; Shabbir, M.A.B.; et al. Aqueous two-phase system (ATPS): An overview and advances in its applications. Biol. Proced. Online 2016, 18, 18. [CrossRef]

21. Qi, L.; Xu, Z.; Jiang, X.; Hu, C.; Zou, X. Preparation and antibacterial activity of chitosan nanoparticles. Carbohydr. Res. 2004, 339, 2693-2700. [CrossRef] [PubMed]

22. Liu, X.; Pai, P.J.; Zhang, W.; Hu, Y.; Dong, X.; Qian, P.Y.; Chen, D.; Lam, H. Proteomic response of methicillin-resistant $S$. aureus to a synergistic antibacterial drug combination: A novel erythromycin derivative and oxacillin. Sci. Rep. 2016, 6, 19841. [CrossRef] [PubMed]

23. Mu, X.; Yu, H.; Zhang, C.; Chen, X.; Cheng, Z.; Bai, R.; Wu, X.; Yu, Q.; Wu, C.; Diao, Y. Nano-porous nitrocellulose liquid bandage modulates cell and cytokine response and accelerates cutaneous wound healing in a mouse model. Carbohyd. Polym. 2016, 136, 618-629. [CrossRef] [PubMed]

Sample Availability: Samples of the compounds are not available from the authors.

(C) 2020 by the authors. Licensee MDPI, Basel, Switzerland. This article is an open access article distributed under the terms and conditions of the Creative Commons Attribution (CC BY) license (http://creativecommons.org/licenses/by/4.0/). 